\title{
Correction to: Extra energy for hearts with a genetic defect: ENERGY trial
}

\author{
B. O. van Driel · A. C. van Rossum · M. Michels · R. Huurman · J. van der Velden
}

Published online: 20 March 2019

(c) The Author(s) 2019

\section{Correction to:}

Neth Heart J 2019

https://doi.org/10.1007/s12471-019-1239-0

In the version of the article originally published online, there was an error in Fig. 1a. In the $3 \times 3$ panel, the images indicated as 'CMR cine of four-chamber view', 'Parametric image of k2' and 'Polar map of k2' were incorrectly matched to the rows indicated as Control, MYBPC3 carrier and MYH7 carrier. The im- age should have shown the lowest $\mathrm{O} 2$ consumption (most purple) images for Control and highest O2 consumption (most orange) for MYH7 carriers. The corrected figure is given below.

Open Access This article is distributed under the terms of the Creative Commons Attribution 4.0 International License (http://creativecommons.org/licenses/by/4.0/), which permits unrestricted use, distribution, and reproduction in any medium, provided you give appropriate credit to the original author(s) and the source, provide a link to the Creative Commons license, and indicate if changes were made.

The online version of the original article can be found under https://doi.org/10.1007/s12471-019-1239-0.

B. O. van Driel $(\varangle) \cdot$ J. van der Velden

Department of Physiology, Amsterdam Cardiovascular

Sciences, Amsterdam UMC, location VUmc, Amsterdam,

The Netherlands

b.vandriel@vumc.nl

A. C. van Rossum

Department of Cardiology, Amsterdam UMC, location

VUmc, Amsterdam, The Netherlands

M. Michels $\cdot$ R. Huurman

Department of Cardiology, Erasmus Medical Center

Rotterdam, Rotterdam, The Netherlands 
Fig. 1 The ratio between external work and myocardial oxygen consumption to obtain myocardial external efficiency was determined in 28 asymptomatic mutation carriers (14 MYBPC3 and 14 MYH7), 10 manifest $\mathrm{HCM}$ patients and 14 healthy controls using $\left[{ }^{11} \mathrm{C}\right]$-acetate positron emission tomography (PET) and cardiovascular magnetic resonance imaging (CMR). a CMR-derived cardiac 4-chamber view and parametric images of $\left[{ }^{11} \mathrm{C}\right]-$ acetate PET derived $k 2$ with corresponding polar maps. As can be seen clearly, oxygen metabolism was higher in asymptomatic mutation carriers compared to controls [7]. b Myocardial efficiency is not further reduced in advanced HCM patients compared with mutation carriers [8]

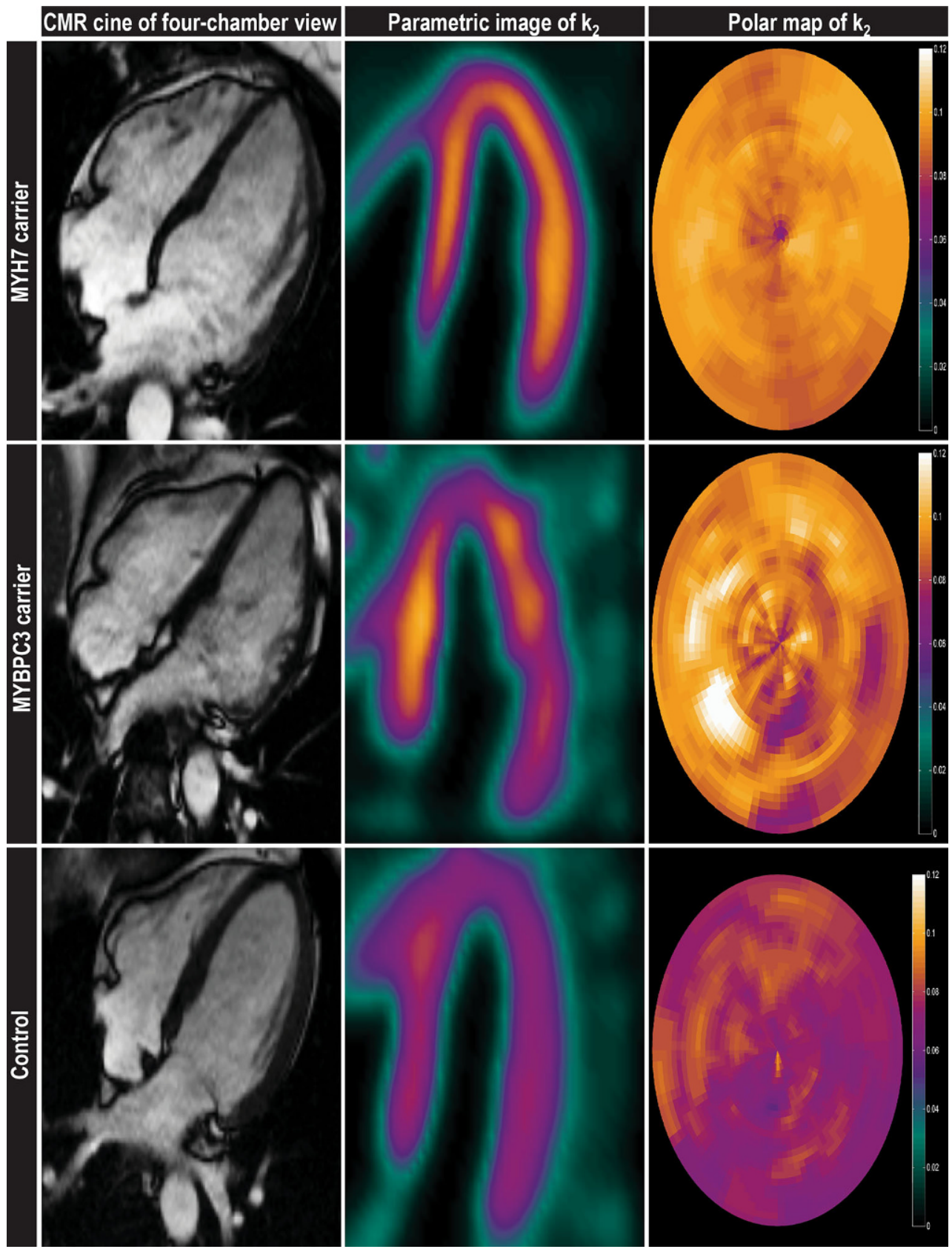

